\title{
Development of reference test specimens for the standardisation of active thermography with flash excitation
}

by Ch. Maierhofer*, M. Reischel*, H. Steinfurth*, M. Röllig*, P. Myrach* and M. Ziegler*

${ }^{\star}$ BAM Federal Institute for Materials Research and Testing, Division 8.4, Unter den Eichen 87, 12205 Berlin, Germany, christiane.maierhofer@bam.de

\begin{abstract}
In this paper, results of a project concerning the development of a testing standard for active thermography with flash excitation are presented. In addition to the standardisation of the appropriate selection of equipment and procedures for testing and data analysis, the standard will describe suitable reference test specimens. These test specimens can be used for the qualification of the equipment as well as for the validation of the method including data processing concerning different areas of application.
\end{abstract}

\section{Introduction}

For non-destructive testing in industry, active thermography methods are applied more and more often for quality assurance, diagnosis, and condition monitoring. Existing thermography standards describe the general principles, equipment, and terms [1-4] or are related to a very limited area of application, e. g. to aerospace structures or thickness measurements $[5,6]$. But for a reliable and reproducible use of active thermography with flash excitation, also a practical testing standard is urgently required. Currently, for each area of application flash thermography has to be validated by extensive tests and calibration procedures. Comprehensive testing instructions have to be developed for each implementation individually. Thus a new national standard which is intended as a European standard in the near future will support industry and SMEs to perform standardized testing. Further on, the acceptance of the application of flash thermography in research and industry will increase, thus market opportunities for device manufacturers and service providers will improve. For supporting this process, BAM and further subcontractors are working on a project titled Development of standards for active thermography with flash excitation. This project is supported by the Deutsches Institut für Normung e. V. (DIN) and the Federal Ministry of Economics and Technology (BMWi), project administrator is the German Aerospace Center (DLR).

For active thermography, very often flash excitation is applied as a fast, efficient and multifrequency energy source. Flash excitation is very well suited for the detection and quantification of defects close to the surface and with an orientation parallel to the surface. With the short optical impulse, a very thin layer close to the surface is heated up. Afterwards, the heat is transported into the structure by thermal conductance, but also heat losses due to thermal radiation and convection occur. The total penetration depth and thus the detectability of defects of course depends on the absorbed heat, the material system, its thermal properties, defect geometry etc., but usually this technique is suited to detect defects in a few millimetres depth. As sources, Xenon flash lamps are applied providing light pulses which are converting energies up to $10 \mathrm{~kJ}$. The excitation energy can be increased by combining multiple lamps. The energy also depends on impulse duration, which usually varies between 1 and $5 \mathrm{~ms}$, but cannot be easily modified within one system. Less impulse widths imply a reduced radiated energy. The radiated spectrum can be compared to that of a black body emitting at $5000 \mathrm{~K}$. Beam shaping can be performed by suitable reflectors. For avoiding an additional heating of the test objects due to the residual heat of the lamps, the lamps can be protected by Plexiglas sheets. Depending on the frequency of testing, the flash lamps including reflector and Plexiglas sheet can be cooled by air or water. Although flash lamps are the most efficient sources for impulse heating with very short impulses, in principle this is also possible by using LED arrays or lasers. Here, the main advantages are a limited bandwidth of the radiation which does not overlap with the wavelength range of the IR camera and a much less residual heat after switching off the source. The disadvantage is the limited energy provided by these systems for testing larger areas.

Data processing can be classified to preprocessing, signal and image processing and inversion techniques. The main aim of signal and image processing as well as of inversion is to obtain quantitative information about material and defect parameters. Currently, there exists a large amount of techniques for signal and image processing, the most relevant ones to be considered in a future standard are described in chapter 4 . Data inversion is still under development, see for example $[7,8]$.

Flash thermography is already applied in industry for testing of gas turbine blades [9]. A good overview about applications in automotive industry is given in [10] and there are further opportunities for testing of spot welded joints [11]. For the development of standards for flash thermography, systematic investigations concerning limits of application, detectability and measuring inaccuracy are still lacking. This includes the characterisation of suited equipment, as well as the consideration of the demands of the users. Therefore, typical testing problems concerning structural elements made of different materials and geometries have been collected and specified in close collaboration with industry. From these 
specifications, test specimens have been developed and are still under optimisation. These might be used as reference test specimen in the near future. These structures as well as testing results will be presented in this contribution.

\section{State of the art in standardisation of active thermography}

As active thermography is a relatively new but very promising non-destructive testing method, the increase of application of this method to different areas of application in research and industry for quality assurance, damage diagnosis and condition monitoring has started quite recently. Thus, until now there have been only a few activities on standardisation.

In Germany, at DIN e. V. first a standard on General Principles of thermographic testing in NDT was established in 2004 [1], followed by a standard on Equipment in 2005 [2], which was updated in 2011, and a standard on Terms and Definitions in 2006 [3]. In 2009, the first test standard on Thermographic testing of electric installations was published [12] followed in 2010 by a standard on Active thermography [4].

Four of the above mentioned standards have been translated to English (DIN 54190 Part 1 to Part 3, DIN 54192) and have been submitted to the new Working Group WG11 Infrared Thermographic Testing of CEN TC 138. The first meeting of the TC took place at Berlin in September 2011. The activities started with the development on a European standard on General Principles.

At the international level, the ISO TC 135 Non-destructive testing has published a draft standard on Vocabulary in ISO/FDIS 10878:2011 [13]. Here, up to 186 terms are listed describing optics, detection of infrared radiation, temperature measurement, thermography and active thermography. Further ISO standards are planned, which have to be aligned with CEN activities.

The concrete application of flash thermography for testing composites is described in ASTM E 2582-07 Standard Practice for Infrared Flash Thermography of Composite Panels and Repair Patches Used in Aerospace Applications [5]. NDT of delaminations at bridge decks is appointed in ASTM D 4788 [14]. Here, sun radiation is used for active heating. For the determination of layer thickness using thermal waves, a method based on the periodic heating of surfaces is given in EN 15042-2 Thickness measurement of coatings and characterization of surfaces with surface waves - Part 2: Guide to the thickness measurement of coatings by photothermic method, which was developed in 2006 by CEN TC 262 Metallic and other organic coatings [6]. But here, as an area of application, only the measurement of layer thickness is considered. Imaging systems like infrared cameras are not regarded for the detection of infrared radiation.

In July 2012, the publication of EN ISO 9712:2012 Non-destructive testing - Qualification and certification of NDT personnel - General principles is planned [15], which is also setting the specifications for training in thermography. In DIN EN 473, which will be displaced by ISO 9712, this method was not included. Thus, a high level of education in thermography applied to NDT will be guaranteed for the future.

But for active thermography, still standards are required, which describe the areas of application, equipment, measurement uncertainties, influence quantities and limits for different thermal excitations like flash thermography and lock-in thermography. And currently, there are no general descriptions of reference test specimens which can be used for controlling the specifications of method and equipment.

\section{Reference test specimen}

Considering the standards described above, only in ASTM E 2582-07 [5] the application of reference test specimen is advised with a rough description of its design. Here, first a detectability standard including five known flaws representing the range of aspect ratio to be expected for testing in the composite material should be provided. These can also be replaced by flat bottom holes. Second, a uniformity standard should help to control the uniformity of the light distribution radiated by the flash lamps. This uniformity standard could be represented by an aluminium plate. In other cases, usually flat bottom holes and step wedges are used. Further reference test specimen for fibre reinforced composites and aerospace components (honeycomb structure) specimen are described in [16] and [17], respectively. Here, more complex structures including flat bottom holes, impact damages, delaminations, cells filled with water etc. have been designed.

In the frame of the research projects presented here, from cooperation with the industry and discussions during a national workshop on the application of flash thermography, the following structures and materials have been extracted to be considered for reference test specimens:

- Join connections and coatings of and between metals with different thermal diffusivities

- $\quad$ Damage and inhomogeneities inside CFRP and GFRP structures

- Delaminations and structures of ceramic coating (e. g. thermal barrier coating)

- Inhomogeneities inside ceramics and building materials

\subsection{Metal join connections}

Flash thermography is very well suited for testing joined connections of thin metal sheets, like laser welded joints, spotwelded joints, solder joints and adhesive joints. In some cases, the interpretation of the results is not straightforward as a good thermal contact does not necessarily imply a perfect mechanical contact. For the fabrication of reference test specimens comprising artificial delaminations, these delaminations should be realized reproducible. 
First, solder connections of copper and of steel sheets have been realized using solder preforms with open areas where these areas were filled with a thermal stable foil of low thermal conductance [18]. In figure 1 left, a sketch and a photo of the geometry of these test specimens are shown, which have been realised for plate thicknesses of 2 and $3 \mathrm{~mm}$. The area of the largest delamination is $10 \times 10 \mathrm{~mm}^{2}$ and of the smallest is $3 \times 3 \mathrm{~mm}^{2}$. As a reference method, the ultrasonic immersion technique was applied by CERN and the C-scans of the relevant depths will be compared to the thermograms.

Second, a steel test specimen with a very flat and polished surface was constructed for realising adhesive joints directly between metal interfaces without glue, see figure 1 right. At the surface of the bottom metal piece, deepenings with a depth of $0.3 \mathrm{~mm}$ and sizes between $10 \times 10$ and $2 \times 2 \mathrm{~mm}^{2}$ have been included with spark erosion. Plates with different thicknesses between 0.5 and $3 \mathrm{~mm}$ have been used as top sheet. The top sheets can be fixed to the underlying block by counter-sunk screws.
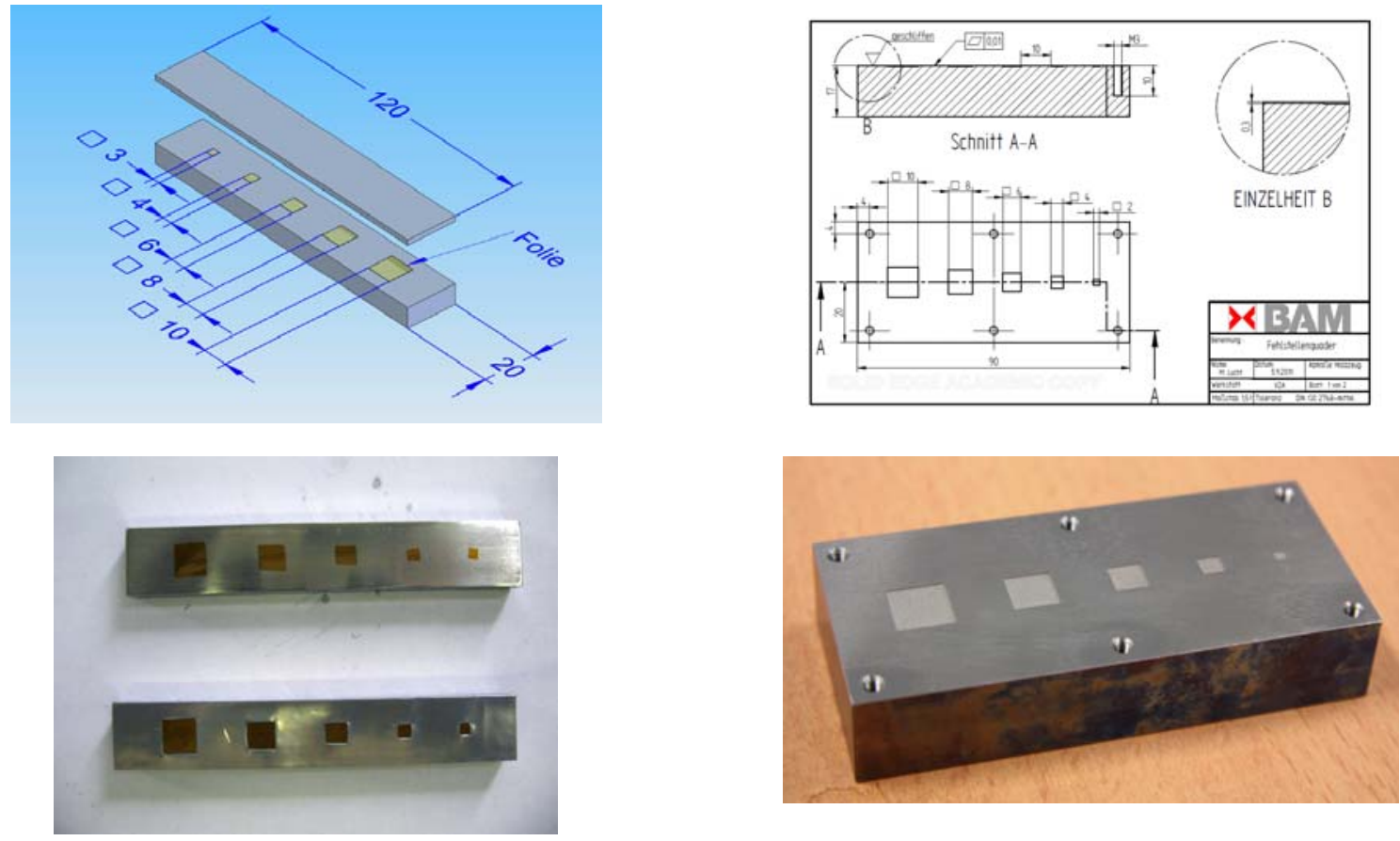

Figure 1: Left: Sketch (top) and photo (bottom) of the solder test specimen before soldering. Right: Sketch (top) and photo of steel test specimen with eroded deepenings before mounting of the top shield (bottom) for realising an adhesive joint. Dimensions are given in $\mathrm{mm}$.

\subsection{CFRP structures}

The field of application of fibre reinforced composites is manifold and ranges from simple technical applications to highperformance structures in energy sector and aerospace. Defects and inhomogeneities already occur during production or during use due to external damage, load or fatigue. These include:

- Ondulations of fibres

- Inhomogeneous distribution of fibres and fibre orientation

- $\quad$ Pores, larger voids, or variations of the density of the matrix

- Detachment of the matrix and delaminations

- Cracks of fibres

The thermal properties are mainly determined by the fibres, which might be very anisotropic. Thus, the distribution of heat inside the structures depends very much on the used orientation and combination of fibres. Nevertheless, flash thermography has already been proven to be very well suited for investigating these structures. As already described above, for testing of thin plates and of repair patches of CFRP, an ASTM standard has been issued [5]. Within the project, different test specimens have been developed containing artificial delaminations as well as defined impact damages.

Artificial delaminations with different sizes have been included at different depth inside two step test specimen, see figure 2. The delaminations have been realised with two folded sheets of Polytetrafluoroethylene (PTFE) with a thickness of $0.1 \mathrm{~mm}$ each and with sizes between $20 \times 20$ down to $2 \times 2 \mathrm{~mm}^{2}$. Each of the step test specimen consists of 5 steps, thus the covering of the delaminations varied between 0.5 and $5 \mathrm{~mm}$ with steps of $0.5 \mathrm{~mm}$. As material, Epoxy resin impregnated carbon filamentary materials (prepregs) with a thickness of the layers of $0.13 \mathrm{~mm}$ have been used. 
For the samples with impact damage, first plates have been constructed according to DIN 65561 [19] containing 24 and 36 layers ordered symmetrically to the mid layer. The size of the plates was $150 \times 100 \times 3 \mathrm{~mm}^{3}$ and $150 \times 100 \times$ $4.7 \mathrm{~mm}^{3}$. Impact damages have been introduced by a falling weight with two different impactors with a radius of 8 and $10 \mathrm{~mm}$ and weights of 58.6 and $61.9 \mathrm{~g}$, respectively. These have been mounted onto two different slides. In total, impact energies between 4 and $25 \mathrm{~J}$ have been realized. As a reference method, the samples with impact damage have been investigated with computer tomography (CT).
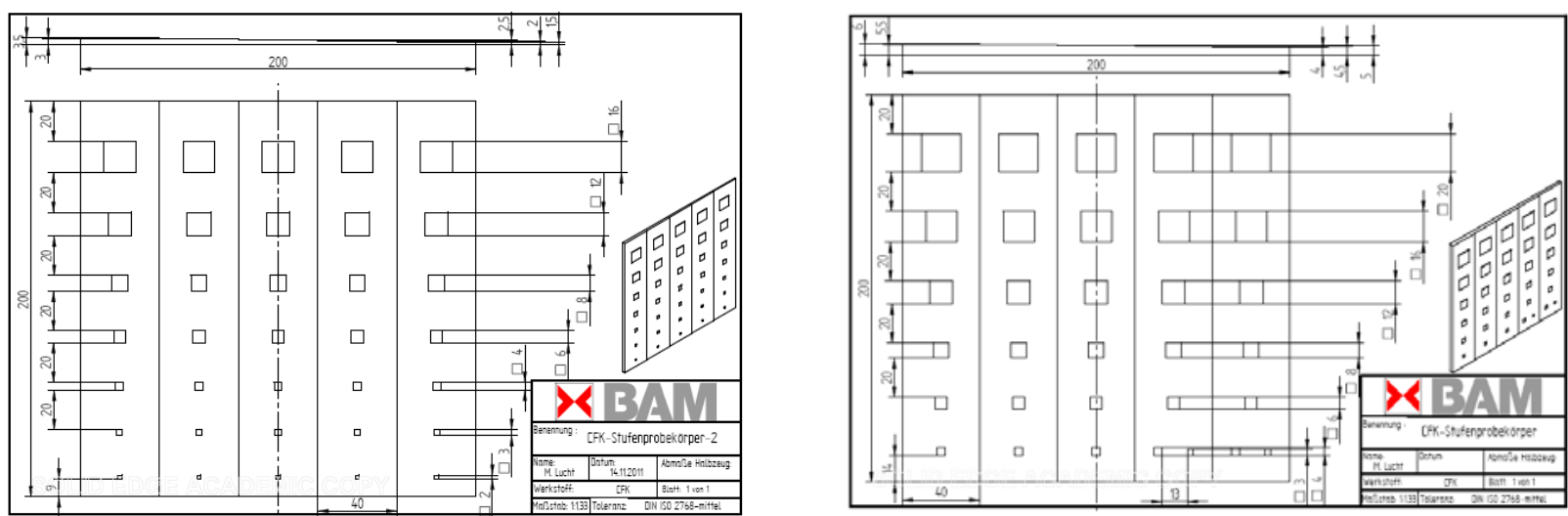

Figure 2: Sketches of CFRP step test specimen with artificial delaminations having different sizes and depth. Left: Variation of depth between 0.5 and $2.5 \mathrm{~mm}$, and of sizes between $16 \times 16$ and $2 \times 2 \mathrm{~mm}^{2}$. Right: Variation of depth between 3 and $5 \mathrm{~mm}$, and of sizes between $20 \times 20$ and $3 \times 3 \mathrm{~mm}^{2}$. Dimensions are given in $\mathrm{mm}$.

\section{Characterisation of technical equipment and data recording}

The thermal data of the above described test specimen have been generated with a SMWIR infrared (IR) camera with a cooled InSb FPA detector with $512 \times 640$ pixels being sensitive between 1.2 and $5.4 \mu \mathrm{m}$. The frames have been recorded in the integrate-than-read snapshot modus with a frame rate of $93 \mathrm{~Hz}$. A SMWIR lens with a field of view of $15.1^{\circ} \times 18.8^{\circ}$ was used together with a temperature controlled band pass filter being transparent between 3.7 and $5.3 \mu \mathrm{m}$.
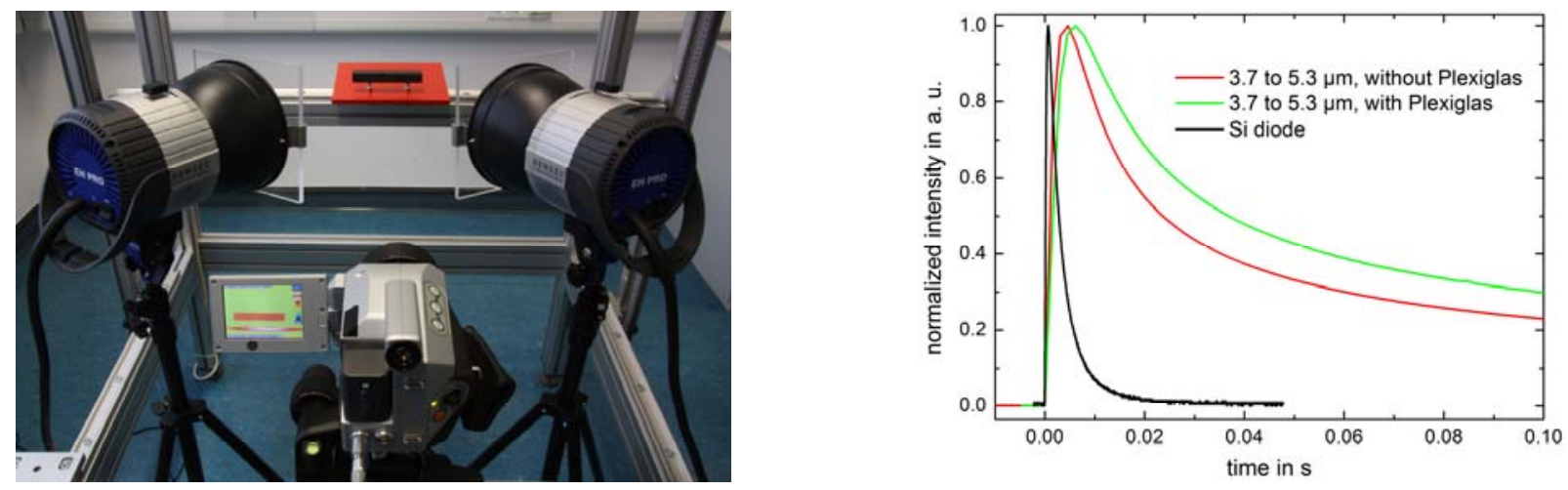

Figure 3: Left: Experimental set-up for performing measurements with flash excitation with two flash lamps. (Here, a microbolometer IR camera is shown). Right: Temporal behaviour of the impulse of the flash lamp measured in the visible and NIR range directly with a Si diode (black). The thermal response of a black paper was measured with the IR camera in the range between 3.7 to $5.3 \mu \mathrm{m}$ without (red) and with (green) Plexiglas sheet.

For thermal excitation, two to four flash lamps with an energy consumption of $6 \mathrm{~kJ}$ each have been mounted in optimum distance to the test samples realising a homogeneous and efficient heating, but not disturbing data recording with the camera, see figure 3, left. A comparison of four different flash lamps showed that although the radiated intensity slightly varies, the shape of the impulse is similar. For reducing sample heating from residual heat of the lamps, Plexiglas sheets haven been mounted in front of the lamps. In figure 3 right, the impulse emitted from one flash lamp is shown measured with a Si diode without Plexiglas sheet in the visible and NIR range. Here, the Si diode was positioned opposite to the camera and several grey filters have been applied preventing an overload of the sensor. The other data 
in figure 3 right have been recorded with the IR camera with and without Plexiglas sheet. Here, the thermal response of a thin black paper sheet was recorded in sub-windows modus with a frame rate of $1 \mathrm{kHz}$. All signals have been normalized. In the visible range, the duration of the flash is about $2.6 \mathrm{~ms}$ (FWHM). Those measured with the IR camera are considerably longer, but it is assumed that the thermal response time of the paper cannot be neglected in relation to the pulse duration. Additionally, it is shown that the impulse of the lamp measured between 3.7 to $5.3 \mu \mathrm{m}$ is much slower and even decreases with the Plexiglas sheet (although the intensity of the thermal response detected with the Plexiglas sheet is considerably lower) as in the visible range. Therefore, it is planned to use cooled plates. For the characterisation of equipment, this behaviour has to be considered, e. g. when comparing the experiment to numerical simulations.

For further details of the characterisation of the flash lamps, see another contribution of BAM to these proceedings titled Determination of the spatial energy distribution generated by means of a flash lamp by $\mathrm{R}$. Krankenhagen et al..

\section{Data analysis}

For the analysis of data recorded with flash thermography, several methods have been developed and applied during the last years considering either only single thermograms or complete sequences of thermal images, sometimes including also the thermal flash excitation. Good overviews are given in [20, 21, 22]. The main aim of these methods is to get as far as possible quantitative information about the structure and defect under investigation from the thermal and temporal response after thermal excitation. E. g., these methods give information about the thermal properties of the surrounding structure and the defect, about the lateral size and depth of the defect and/or about the thickness of layers or structures. Among these, the following methods have achieved acceptance to a broader user domain:

i. Thermal contrast: The thermal contrast of the defect area is calculated by subtracting the temperature-time curve of a sound area from the temperature-time curve of each pixel. Thus, for a specific defect usually a maximum thermal contrast $\Delta T_{\max }$ occurs at a distinct time $t_{\max }$ [20]. Both values can be used for generating calibration curves for various kinds of defects. A visualisation of a thermogram at $t_{\max }$ gives the best contrast for that specific defect.

ii. TSR (thermal signal reconstruction): Here, for each pixel the logarithmic temperature is plotted as a function of the logarithmic time resulting more or less in a straight line. This line is fitted with a polynomial function of low order which significantly reduces the noise. Afterwards, the first and second order derivatives of the algorithmic temperature verse time function is calculated. Peaks of the second derivative usually mark the time which can be used to calculate the depth of defects or layer thickness (or, if the thickness is known, the thermal properties can be estimated) $[22,23,24]$. Further on, the visualisation of the resulting image along all pixels of the first or second derivatives at this time usually gives a considerably good contrast for the respective defect.

iii. $\quad$ PPT (pulse phase thermography): For each pixel, the temperature-time curve is transformed into the frequency range by fast Fourier Transformation (FFT). Thus, a series of amplitude and phase images can be displayed, which are related to different frequencies $[25,26]$. Depending on material properties and defect depth, in some cases the phase images show an enhanced detectability of defects related to contrast images. This is due to the suppression of disturbing signals which are belonging to other frequencies than the observed ones. Therefore, effects due to inhomogeneous surfaces, inhomogeneous heating or surface reflections can be reduced. In some cases, a further fitting of the thermal sequences using polynomial function can reduce the noise and might improve the results of PPT.

iv. Exponential fit: For each pixel, the temperature-time curve is fitted by a function consisting of either one or two exponential functions. The visualisation of the resulting fit parameters (amplitudes and time constants) can give a better SNR (signal to noise ratio) of the defects as the contrast images [27]. In case of using two exponential functions, the fit is very time consuming.

Depending on the testing problems and recorded data, each method has its advantages and disadvantages which will be discussed below.

\section{Results}

\subsection{Metal join connections}

\subsubsection{Soldered joints}

In figure 4, results of the investigations of the $\mathrm{Cu}$ (left) and steel (right) specimen with soldered joints at depth of $2 \mathrm{~mm}$ (top) and $3 \mathrm{~mm}$ (bottom) are shown. Here, four flash lamps have been used for thermal excitation. 1500 images have been recorded with a frame rate of $93 \mathrm{~Hz}$. For each specimen, the thermogram with maximum thermal contrast and the first or second phase image from PPT are displayed. Additionally, C-scans recorded with ultrasonic immersion technique are shown as a reference.

From these diagrams, the following results are obtained:

- For all specimens, several delaminations with different sizes could be detected by higher surface temperatures. But the position and size of the detected delaminations differ (considerably) from the 
intended values. The delaminations shown in the thermograms and phase images agree very well with those of the ultrasonic data.

- The delaminations at a depth of $2 \mathrm{~mm}$ could be detected with a much better signal-to-noise-ratio (SNR) as those at a depth of $3 \mathrm{~mm}$.

- For the thermograms, at each depth the delamination in Cu and steel could be detected with a similar SNR.

- The phase images related to the steel samples have a much better SNR as those of the Cu samples. For steel, the SNR of the phase images is much better as the SNR of the thermograms. For Cu, the SNR in the phase images slightly decreases in relation to the thermograms.

- As the delaminations differ considerably from the original sketch, only a rough estimation about the spatial resolution is possible. Thus, defects down to a size of $4 \times 4 \mathrm{~mm}^{2}$ might be still detected.

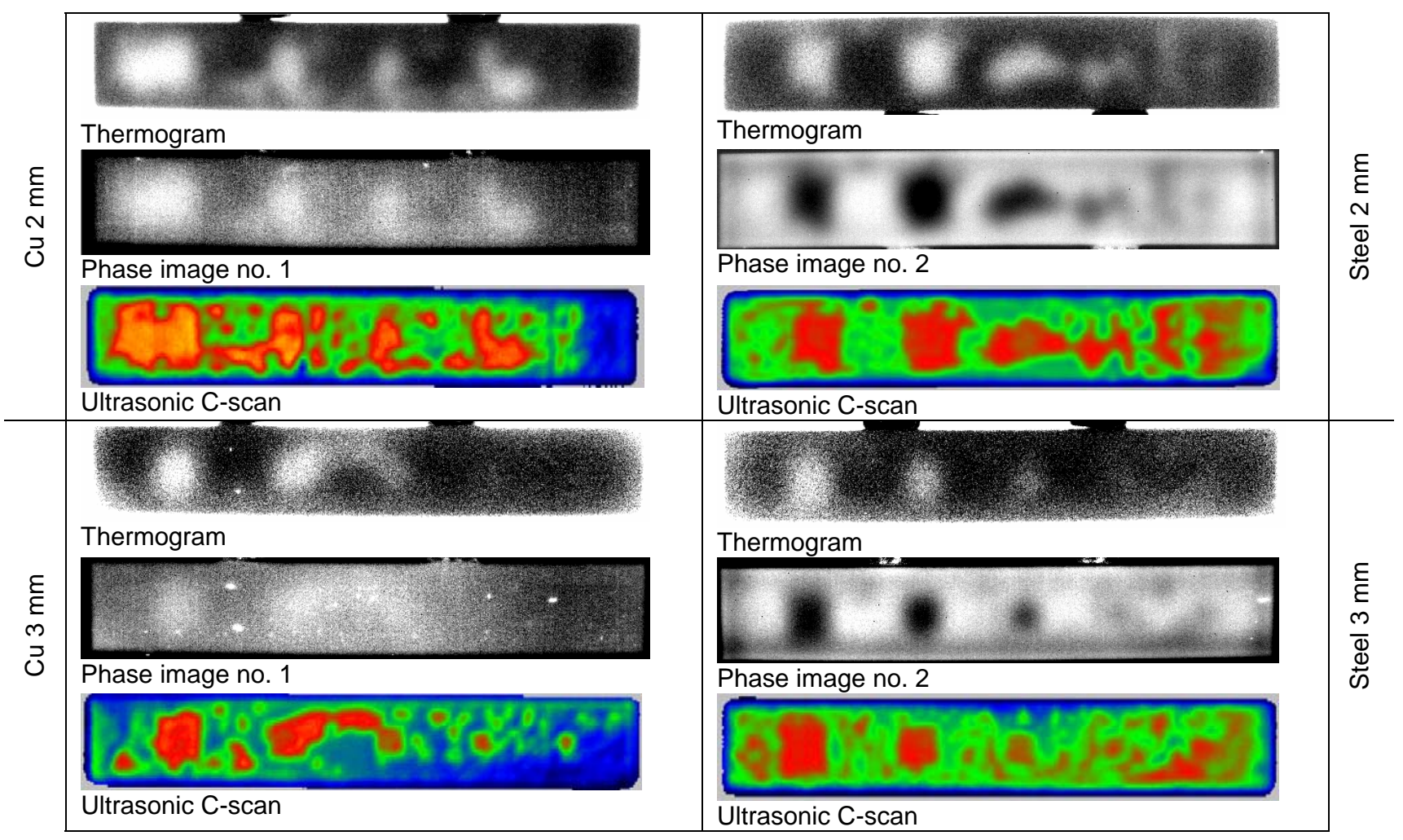

Figure 4: Results of flash thermography investigation of $\mathrm{Cu}$ (left) and steel (right) soldering joints using four flash lamps. For each reference test specimen, the thermogram with maximum thermal contrast of the delaminations and the best phase image are shown. Additionally, the C-scan resulted from ultrasonic immersion investigations provided by CERN are displayed.

\subsubsection{Adhesive joints}

In the following, only selected data of investigations of the $1 \mathrm{~mm}$ and $3 \mathrm{~mm}$ thick steel sheets are presented. In the different columns of figure 5 , the thermograms with maximum thermal contrast, the best phase image and the best image of the first derivative of the following configurations are presented:

- Column 1: Specimen with $1 \mathrm{~mm}$ thick sheet with blank surface (no surface treatment), 4 lamps have been used for thermal excitation

- Column 2: Specimen with $1 \mathrm{~mm}$ thick sheet with treated surface (surface blackening), 4 lamps have been used for thermal excitation

- $\quad$ Column 3: Specimen with $3 \mathrm{~mm}$ thick sheet with treated surface (surface blackening), 4 lamps have been used for thermal excitation

- Column 4: Specimen with $3 \mathrm{~mm}$ thick sheet with treated surface (surface blackening), only 2 lamps have been used for thermal excitation

From these diagrams, the following results are obtained: 
- In all diagrams, the eroded deepenings can be clearly distinguished from the background. Therefore, the adhesion between the bottom block and the covering sheet was large enough to realise a good thermal contact. Nevertheless, the influences of the screws can be clearly seen in all diagrams increasing the thermal contact around them.

- By comparing column 1 and 2, a considerable increase of the SNR is obtained by surface treatment. But already without blackening, defects down to a size of $4 \times 4 \mathrm{~mm}^{2}$ can be detected for a $1 \mathrm{~mm}$ thick steel sheet. With the blackening, also the smallest defect with a size of $2 \times 2 \mathrm{~mm}^{2}$ can be visualized.

- By comparing column 3 and 4 , the use of 4 flash lamps related to only 2 flash lamps with the same energy of each lamp does not significantly increase the SNR.

- $\quad$ For the $3 \mathrm{~mm}$ deepening, the smallest defect which can be detected has a size of $4 \times 4 \mathrm{~mm}^{2}$.

- In the phase image as well as in the image from the first derivative, the defects can be detected with a better SNR and an enhanced spatial resolution as in the thermograms. There is no clear difference between the processed data shown in the phase images and in the first derivative concerning the detectability of the defects.

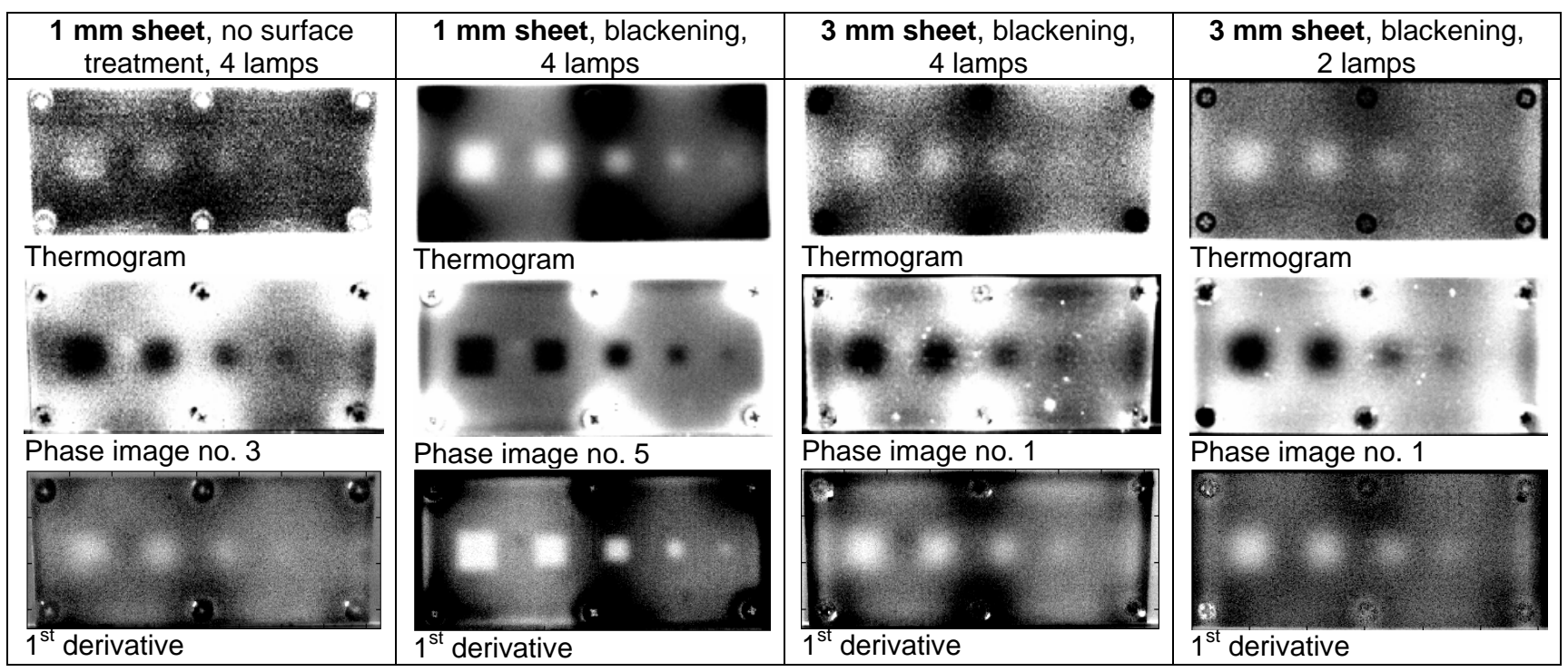

Figure 5: Results of flash thermography investigation of the steel test specimen including eroded deepenings covered with a $1 \mathrm{~mm}$ sheet (left columns) or a $3 \mathrm{~mm}$ sheet (right columns) using four flash lamps or only two (right column). For each data sequence, the thermogram with maximum thermal contrast of the delaminations, the best phase image and the best image of the $1^{\text {st }}$ derivative are shown.

\subsection{CFRP structures}

\subsubsection{Artificial delaminations}

Here, only experimental data recorded at the thinner reference test specimen with steps are shown (maximum thickness: $3.5 \mathrm{~mm}$ ). Four flash lamps have been used for thermal excitation. In figure 6, the thermogram with the maximum thermal contrast (left), the best phase image (middle) as well as the second derivative (TSR, right) are shown. In the thermogram, the delaminations can be detected only for coverings of 0.5 and $1 \mathrm{~mm}$. In the phase image and in the second derivative, also for the $2 \mathrm{~mm}$ covering defects down to a size of $8 \times 8 \mathrm{~mm}$ can be clearly recognized. The image of the second derivative has a slightly better SNR as that of the phase image.

\subsubsection{Impact damages}

The results of one of the test specimens with an impact damage resulting from an impact with $14.42 \mathrm{~J}$ are displayed in figure 7. Here, only two flash lamps have been applied. A sequence consisting of 1050 frames and a frame rate of $93 \mathrm{~Hz}$ has been recorded. Figure $7 \mathrm{a}$ ) shows a thermogram, which was taken $2.43 \mathrm{~s}$ after the flash. Although at the surface only a small dent is visible, the thermogram shows a much larger area with increased temperature hinting to a lager delamination. The phase images no. 30, no. 8 and no. 2, also shown in figure 7, can be related to different depths. Here, the phase image with the largest number corresponds to the highest frequency and thus to a low information depth. In phase image no. 30, a sharply bounded vertical area is related to a delamination of one of the top layers of the structure. 
In phase image no. 8, the position of the impact can be located clearly with a further damaged area on its left side. Phase image no. 2 shows a larger area which corresponds to even larger delaminations between deeper layers. Thus, the impact results to delaminations between the layers at different depth. The size of the delaminations increases with increasing depth corresponding to the theoretical models of crack mechanics [28].

Thermogram

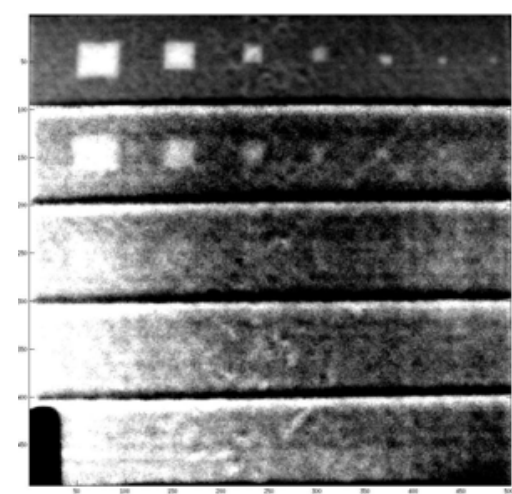

Phase image

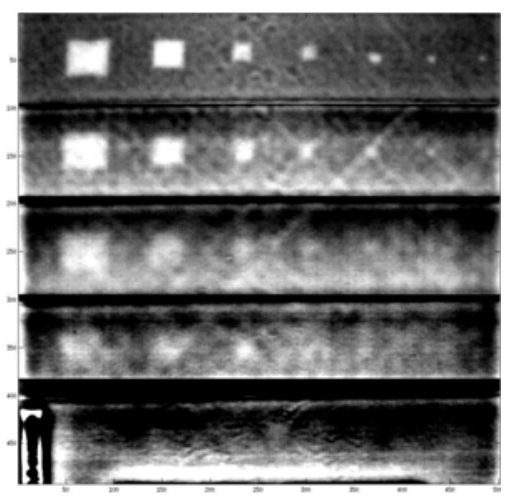

$2^{\text {nd }}$ derivative

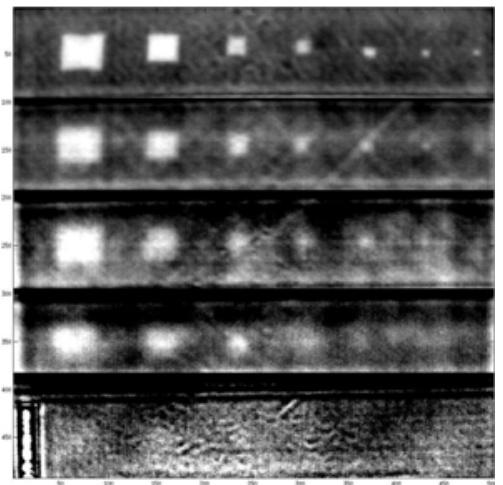

Depth

in $\mathrm{mm}$

0.5

1.0

1.5

2.5

Figure 6: Thermogram (left), phase image (middle) and image of $2^{\text {nd }}$ derivative (TSR, right) of thermal data recorded with 4 flash lamps at the reference test specimen with a total thickness of $3.5 \mathrm{~mm}$. For each step of the specimen, the data in the images have been scaled to minimum and maximum. Sizes of the defects from left to right: $16 \times 16,12 \times 12,8 \times 8,6$ $\times 6,4 \times 4,3 \times 3,2 \times 2 \mathrm{~mm}^{2}$. The depths of the delaminations are shown on the right.

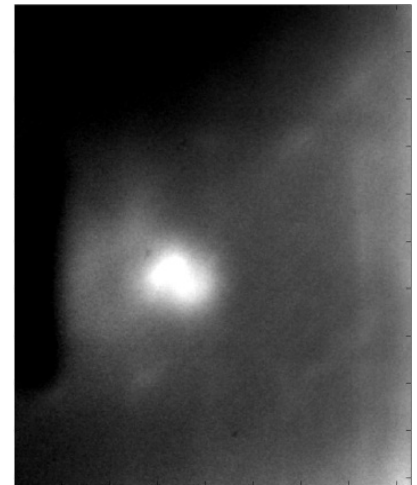

a) Thermogram

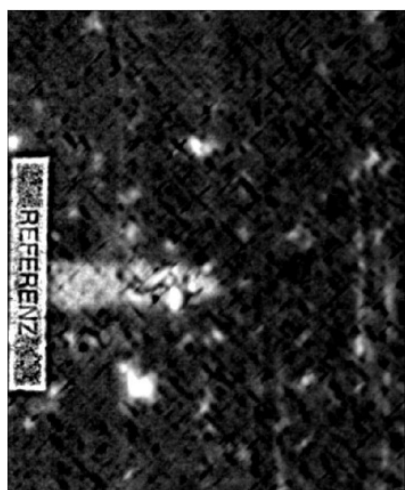

b) Phase image no. 30

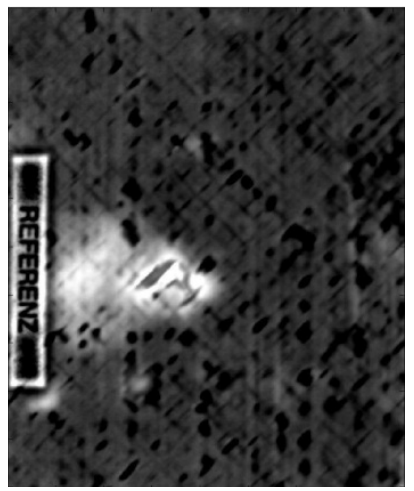

c) Phase image no. 8

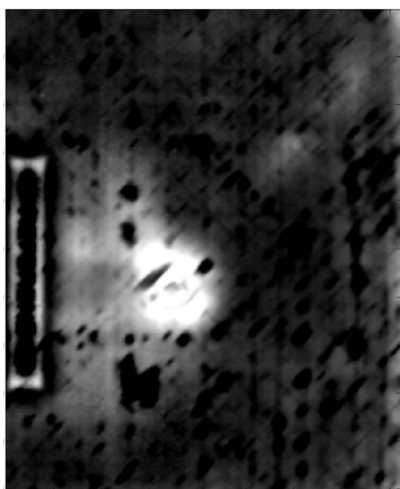

d) Phase image no. 2

Figure 7: Results of investigations at a test specimen with an impact damage of $14.42 \mathrm{~J}$ realized by a falling weight having a diameter of $20 \mathrm{~mm}$, a height of $0.65 \mathrm{~m}$ and a velocity of $3.57 \mathrm{~m} / \mathrm{s}$. a) Thermogram recorded $2.43 \mathrm{~s}$ after the flash. b) Phase image no. 30; c) Phase image no. 8; d) Phase image no. 2. The size of the area shown here is $10 \times 12 \mathrm{~cm}^{2}$.

\section{Summary, discussion and outlook}

For the development of a testing standard defining the application of flash thermography, several reference test specimens have been designed and tested consisting of different materials (copper, steel, CFRP) and including artificial delaminations as well as delaminations induced by impact damages (only for CFRP). These reference test specimens enable the quantification of the detection limits of a given experimental set-up concerning the maximum penetration depth and the spatial resolution. The given experimental set-up here consisted of the excitation sources (here: two to four flash lamps) and the IR camera. Further on, the experimental data recorded at these test specimens can be used for assessing different methods for data processing. Here, thermograms with the highest thermal contrast have been compared to phase images obtained by pulse phase thermography and to the first and second derivative obtained by 
TSR. In summary, the following results have been obtained concerning the detection limits and assessment of data analysis methods:

- The smallest defects investigated here had a size of $2 \times 2 \mathrm{~mm}^{2}$. These could be detected down to a depth (coverage) of $1 \mathrm{~mm}$ in CFRP and of $2 \mathrm{~mm}$ in steel with surface blackening.

- $\quad$ Not in all cases an increase of radiated intensity (here: by using four flash lamps instead of two) lead to an increase of SNR of the defects

- Only for the materials with lower thermal diffusivity, data processing using PPT lead to an increased SNR, e. g. for steel and CFRP. Due to the high thermal diffusivity of $\mathrm{Cu}$, the cooling down occurred very fast, thus the defect information was only present in a few thermal images and PPT does not resulted in an enhanced SNR.

- There is no clear advantage of either using PPT or TSR for data processing. Here, further quantitative investigations are planned.

As reference test specimen, it is shown that the specimen with the eroded deepenings seems to be very suitable as it can be constructed reproducible and different coverings can be easily realised by exchanging the covering plates. It only has to be considered that the surfaces of the material at the interface have to be very smooth and that the tightening of the screws is uniform and enables a good thermal contact. For future tests, it is planned to construct a similar specimen using $\mathrm{Cu}$ and other materials.

Further reference test specimens are under construction:

- $\quad$ Test specimen made of ceramics by using a 3D printer (rapid prototyping)

- $\quad$ Steel test specimen with ceramic coating containing artificial delaminations

\section{Acknowledgement}

This project titled Development of standards for active thermography with flash excitation was funded in the frame of the research programme Innovation with Norms and Standards by the Federal Ministry of Economics and Technology in cooperation with DIN e. V.. We appreciate the support of Ch. Scheuerlein from CERN for the construction of the test specimen consisting the soldering joints. The CFRP test specimens have been constructed by J. Häberle and J. Schulz from ZFL Zentrum für Faserverbunde und Leichtbau Haldensleben. P. Wossidlo from BAM 9.1 supported us with the equipment for generating the impact damages.

\section{REFERENCES}

[1] DIN 54190-1:2004-08, Non-destructive testing - Thermographic testing - Part 1: General principles

[2] DIN 54190-2:2011-09, Non-destructive testing - Thermographic testing - Part 2: Equipment

[3] DIN 54190-3:2006-02, Non-destructive testing - Thermographic testing - Part 3: Terms and definitions

[4] DIN 54192:2010-11, Non-destructive testing - Active Thermography

[5] ASTM E 2582-07, Standard Practice for Infrared Flash Thermography of Composite Panels and Repair Patches Used in Aerospace Applications

[6] EN 15042-2:2006-06, Thickness measurement of coatings and characterization of surfaces with surface waves - Part 2: Guide to the thickness measurement of coatings by photothermic method

[7] Lugin, S., Netzelmann, U.: A defect shape reconstruction algorithm for pulsed thermography. NDT\&E International Vol. 40, pp. 220-228, 2007

[8] Richter, R., Maierhofer, Ch., Kreutzbruck, M., Schilling, M.: Numerical method of active thermography for the reconstruction of back wall geometry. In: Proceeding of 6th Workshop 'NDT in progress', Czech society for nondestructive testing (Cndt), pp. 245-252, 2011

[9] Goldammer, M. et al.: Automatisiertes System zur thermographischen Prüfung von Gasturbinenschaufeln. DGZfP-Jahrestagung 2003, Mainz

[10] Siemer, U.: Einsatz der Thermografie als zerstörungsfreies Prüfverfahren in der Automobilindustrie. Dissertation, Saarbrücken, 2010, http://scidok.sulb.unisaarland.de/volltexte/2010/3062/pdf/Dissertation_UlrikeSiemer_16.03.2010.pdf

[11] Schlichting, J., Brauser, S., Pepke, L.-A., Maierhofer, Ch., Rethmeier, M., Kreutzbruck, M.: Thermographic testing of spot welds. NDT \& E International, Vol. 48, pp. 23-29, 2012

[12] DIN 54191:2009-03, Non-destructive testing - Thermographic testing of electric installations

[13] ISO/FDIS 10878:2011, Non-destructive testing - Infrared thermography - Vocabulary

[14] ASTM D 4788:2003, reapproved 2007, Standard test method for detecting delaminations in bridge decks using infrared thermography

[15] EN ISO 9712:2012, Non-destructive testing - Qualification and certification of NDT personnel - General principles

[16] Avdelidis, N.P. Maldague, X.P.V. et al.: NDT characterisation of carbon-fibre and glass-fibre composites using non-invasive imaging techniques. In: Proc. of QIRT 2010, Quebec 
[17] Ibarra-Castanedo, C. Maldague, X.P.V. et al.: Recent progresses in the inspection of aerospace components by infrared thermography. In: 17th World Conference on Nondestructive Testing, 25-28 Oct 2008, Shanghai, China

[18] Maierhofer, Ch., Röllig, M., Steinfurth, H., Ziegler, M., Heck, S.J., Scheuerlein, C.: Aktive Thermografie zur zerstörungsfreien Prüfung von Lötverbindungen. In: Proceedings DGZfP-Jahrestagung 2011, DGZfP BB 127, 2011

[19] DIN 65561:1991, Aerospace; fiber reinforced plastics; testing of multidirectional laminates; determination of compressive strength after impact test.

[20] Maldague, X.: Theory and Practice of Infrared Technology for Non-destructive Testing. John Wiley and Sons, 684 p., 2001

[21] Ibarra-Castanedo, C., Piau, J.-M., Guibert, S., Avdelidis, N. P., Genest, M., Bendada, A., Maldague, X. P. V.: Comparative Study of Active Thermography Techniques for the Nondestructive Evaluation of Honeycomb Structures. Research in Nondestructive Evaluation, 20:1, pp. 1-31, 2009

[22] Omar, M. A., and Zhou, Y.: A quantitative review of three flash thermography processing routines. Infrared Physics and Technology 51(4), pp. 300-306, 2008

[23] Shepard, S.: Flash thermography of Aerospace Composites. In: Proc. of IV Conference Panamericana de END Buenos Aires, 2007

[24] Balageas, D. L.: Personal thoughts on the occasion of the Xth QIRT Conference. Presentation at 10th QIRT Conference - Quebec, July 2010, http://qirt.gel.ulaval.ca/archives/qirt2010/papers/QIRT\%202010-161.pdf

[25] Maldague, X., Marinetti, S.: Pulse Phase Infrared Thermography. J. Appl. Phys. 79, pp. 2694-2698, 1996

[26] Maldague, X., Galmiche, F., Ziadi, A.: Advances in pulsed thermography. Infrared Physics and Technology 43 pp. 175-181, 2002

[27] Maierhofer, Ch., Brink, A., Röllig, M., Wiggenhauser, H.: Detection of shallow voids in concrete structures with impulse thermography and radar. NDT \& E International Vol. 36, pp. 257-263, 2003

[28] Ehrenstein, G.: Faserverbund-Kunststoffe - Werkstoffe - Verarbeitung - Eigenschaften. 2nd Edition, Carl Hanser Verlag München Wien, pp. 297, 2006 